\title{
Genetic Causation of Transgender Neruobiological Identity, An Annotated Bibliography.
}

\author{
Hontas Farmer ${ }^{1}$ \\ ${ }^{1}$ Affiliation not available
}

January 24, 2022

$\boldsymbol{R}$ ights are not decided by science but by law and laws are made by man. However, in this scientific age it has been decided that people who are members of a minority by nature generally have the same rights as anyone else. This is known as biological essentialism. Most transgender activist do not accept this, for the sake of discussion let us accept it. Therefore, the question of transgender rights comes down to how science answers the following questions.

Do transgender people have the choice of completely conforming to gender norms and just being manly men or feminine women?

Does the available data show that transgender people exist due to genetic, hormonal, neurological and or any other physical and immutable cause?

Does hormone therapy for enough time or given early enough in life level the playing field for sports, and what variables effect this?

What are the broader human rights implications of the answers to the above?

- Multiple independent studies have demonstrated a high probability that mutations to the Androgen Receptor (AR) and estrogen receptor genes ER $\beta$ (Fernández et al., 2018) (Theisen et al., 2019)

- These genetic differences correspond to various differences in brain strucutre such that sexually dimorphic brain structures of transwomen match those of ciswomen, and those of transmen match those of cismen. (Boucher \& Chinnah, 2020)

- The differences are also mannifested in the form of reduced fertility and general undermasculinization even prior to any hormone treatment. (Amir et al., 2022)

- The above finding is backed by MULTIPLE independent expert, peer reviewed, published meta analysis, a study of all available studies. (D'Andrea et al., 2020) Crucially for the issue of fairness in sports there are relevant endocrinological differences. (The Endocrine Socieity, 2020)

- A study done on post pubescent transgender adults by (Hilton \& Lundberg, 2020) concluded a large advantage for transgender women in every sport, however, this article was corrected by the journal due to their undisclosed confict of interest. (Hilton \& Lundberg, 2021)

- A further study, also cited by Hilton as being well done, found after 1-2 years on hormones little to no difference in strength but a 5\% advantage in speed for transwomen. (Roberts et al., 2021)

- Based on (Roberts et al., 2021) the British Journal of Sports Medicine concluded that given the data on competitors who are transitioning as young adults at the elite and collegiate levels there can be a difference that confers advantage, at the same time at the high school, youth and recreational levels when all the data is considered there is not necessarily an advantage. (Ahrendt et al., 2021) 


\title{
Annotated Bibliography with Abstracts
}

\author{
Findings based on Genetic research.
}

Transgenderism is caused by mutations of the androgen and estrogen receiptor genes with effects on brain development, particularly in the basal ganglia. My Notes are in RED any emphasis is mine.

Fernández, Rosa / Guillamon, Antonio / Cortés-Cortés, Joselyn / Gómez-Gil, Esther / Jácome, Amalia / Esteva, Isabel / Almaraz, MariCruz / Mora, Mireia / Aranda, Gloria / Pásaro, Eduardo Molecular basis of Gender Dysphoria: androgen and estrogen receptor interaction2018 Psychoneuroendocrinology , Vol. 98 p. 161-167 https://doi.org/10.1038/s41598-019-53500-y (Fernández et al., 2018)

\begin{abstract}
Background: Polymorphisms in sex steroid receptors have been associated with transsexualism. However, published replication studies have yielded inconsistent findings, possibly because of a limited sample size and/or the heterogeneity of the transsexual population with respect to the onset of dysphoria and sexual orientation. We assessed the role of androgen receptor (AR), estrogen receptors alpha $(\mathrm{ER} \alpha)$ and beta $(\mathrm{ER} \beta)$, and aromatase (CYP19A1) in two large and homogeneous transsexual male-to-female (MtF) and female-to-male (FtM) populations.

Methods: The association of each polymorphism with transsexualism was studied with a twofold subject-control analysis: in a homogeneous population of 549 early onset androphilic MtF transsexuals versus 728 male controls, and 425 gynephilic FtMs versus 599 female controls. Associations and interactions were investigated using binary logistic regression.

Results: Our data show that specific allele and genotype combinations of ER $\beta, \operatorname{ER} \alpha$ and AR are implicated in the genetic basis of transsexualism, and that $M \tau \Phi$ $\gamma \varepsilon \nu \delta \varepsilon \rho \delta \varepsilon \varepsilon \lambda о \pi \mu \varepsilon \nu \tau$

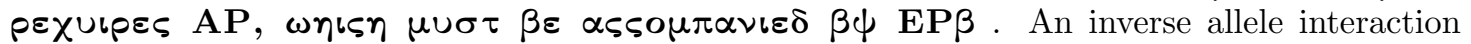
between $\mathrm{ER} \beta$ and $\mathrm{AR}$ is characteristic of the MtF population: when either of these polymorphisms is short, the other is long. ER $\beta$ and ER $\alpha$ are also associated with transsexualism in the FtM population although there was no interaction between the polymorphisms. Our data show that $\mathrm{ER} \beta$ plays a key role in the typical brain differentiation of humans.
\end{abstract}

Theisen, J. Graham / Sundaram, Viji / Filchak, Mary S. / Chorich, Lynn P. / Sullivan, Megan E. / Knight, James / Kim, Hyung-Goo / Layman, Lawrence C. The Use of Whole Exome Sequencing in a Cohort of Transgender Individuals to Identify Rare Genetic Variants 2019-12 Nature Scientific Reports, Vol. 9 p. 20099 https://doi.org/10.1038/s41598-019-53500-y (Theisen et al., 2019)

\begin{abstract}
Approximately $0.5-1.4 \%$ of natal males and $0.2-0.3 \%$ of natal females meet DSM- 5 criteria for gender dysphoria, with many of these individuals self-describing as transgender men or women. Despite recent improvements both in social acceptance of transgender individuals as well as access to gender affirming therapy, progress in both areas has been hampered by poor understanding of the etiology of gender dysphoria. Prior studies have suggested a genetic contribution to gender dysphoria, but previously proposed candidate genes have not yet been verified in follow-up investigation. In this study, we expand on the topic of gender identity genomics by identifying rare variants in genes associated with sexually dimorphic brain development and exploring how they could contribute to gender dysphoria. To accomplish this, we performed whole exome sequencing on the genomic DNA of 13 transgender males and 17 transgender females. Whole exome sequencing revealed 120,582 genetic variants. After filtering, 441 variants in 421 genes remained for further consideration, including 21 nonsense, 28 frameshift, 13 splice-region, and 225 missense variants. Of these, 21 variants in 19 genes were found to have associations with previously described estrogen receptor activated pathways of sexually dimorphic brain development. These variants were confirmed by Sanger Sequencing. Our findings suggest a new avenue for investigation of genes involved in estrogen signaling pathways related to sexually dimorphic brain development and their relationship to gender dysphoria.
\end{abstract}


D'Andrea, Settimio / Pallotti, Francesco / Senofonte, Giulia / Castellini, Chiara / Paoli, Donatella / Lombardo, Francesco / Lenzi, Andrea / Francavilla, Sandro / Francavilla, Felice / Barbonetti, Arcangelo Polymorphic Cytosine-Adenine-Guanine Repeat Length of Androgen Receptor Gene and Gender Incongruence in Trans Women: A Systematic Review and Meta-Analysis of Case-Control Studies 2020 The Journal Of Sexual Medicine, Vol. 17 p. 543-550 https://doi.org/10.1016/j.jsxm. 2019. 12.010 (D'Andrea et al., 2020)

\begin{abstract}
Introduction: It has been hypothesized that gender incongruence in transgender women could result from an antenatal impaired androgen activity on the developing brain. As the length of polymorphic cytosine-adenine-guanine (CAG) repeat sequences in the androgen receptor (AR) gene is inversely correlated with AR transcriptional activity, some studies explored a possible association between long CAG repeats and gender incongruence in trangender women. Yet results remain inconclusive.
\end{abstract}

Aim: To systematically evaluate whether a difference exists in the length of AR CAG repeat sequences between trans women and men without gender incongruence.

Methods: A thorough search of Medline, Scopus, Cochrane Library, Web of Science, and CINAHL databases was carried out to identify suitable case-control studies. Methodological quality of the included articles was assessed using the Newcastle-Ottawa Scale. In the absence of betweenstudies heterogeneity, as assessed by the Cochrane's Q and I2 tests, standardized mean differences (SMDs) in the length of AR CAG repeats were combined using a fixed effect model. Funnel plot and trim-and-fill analysis were used to assess publication bias.

Main outcome measure: The association of gender incongruence in transgender women with longer length of AR CAG repeat sequences was evaluated by calculating pooled standardized mean difference with $95 \%$ confidence interval (CI).

Results: 5 studies included in the quantitative analysis collectively provided information on 795 trans women and 1,355 control men. At the overall estimate, the MtF group exhibited a significantly longer length of AR CAG repeat sequences (pooled standardized mean difference: $0.13,95 \%$ CI: 0.04 to $0.22 ; \mathrm{P}=0.005$; I2 $=0 \%$, Pfor heterogeneity $=0.51$ ). Sensitivity analysis demonstrated the high stability of the result. Funnel plot revealed a possible publication bias, and the trim-and-fill test detected 2 putative missing studies. Nevertheless, the significant association persisted even when pooled estimate was adjusted for publication bias.

Clinical implications: These findings could suggest a contribution of a genetically mediated impairment in androgen signaling in development of gender incongruence for transgender women.

Strength \& limitations: This is the first meta-analysis exploring the relationship between AR $\mathrm{CAG}$ repeat polymorphism and gender incongruence. However, interactions with other functional genetic variants were not explored, and caution should be exercised when generalizing these results because of the possible variability in the distribution of CAG repeats among different populations and ethnic groups.

Conclusion: Trans woman population exhibits significantly longer polymorphic CAG repeat sequences in the AR gene. Further studies are warranted to elucidate whether, how and to what extent multiple functional variants in sex hormone signaling genes could be associated with gender incongruence/dysphoria.

Note: Every scientific study ever has said that further studies are needed for two basic reasons. One is that science is never totally done, though it can be settled beyond any scintilla of doubt, and since performing these studies is our job, we would always like to keep doing it!

Boucher, Ferdinand J. O. / Chinnah, Tudor I. Gender Dysphoria: A Review Investigating the Relationship Between Genetic Influences and Brain Development 2020 Adolescent Health, Medicine and 
Therapeutics, Vol. Volume 11 p. 89-99 https://doi.org/10.2147/ahmt.s259168 (Boucher \& Chinnah, 2020)

Abstract: Gender dysphoria (GD) is a facet of modern human biology which is believed to be derived from the sexual differentiation of the brain. GD "involves a conflict between a person's physical or assigned gender and the gender with which he/she/they identify", as defined in the DSM-5. Individuals report feeling uncomfortable and faced with prejudice from those around them, affecting their mental health. Elucidating the relationship between genetic influences on gonadal and brain development could give an insight into understanding this clinical condition. To explore this issue, a review of the literature database was carried out. Evidence suggests that abnormal biological processes, including mutations in certain genes, can lead to abnormal gonadal development, causing some fetuses to present with indifferent gonads and to be reassigned at birth to the default female sex. This disparity in genetic influences relates to an increased likelihood of a diagnosis of GD. An investigation into complete androgen insensitivity syndrome, involving androgen receptor (AR) gene mutation, suggests that such individuals also experience GD. It is known that the brains of males and females are different. Evidence further suggests that brain anatomy and neuronal signaling pathways are more closely aligned with a person's perceived gender identity. Individuals who present with discordant gonadal and brain developments experience psychological challenges that may contribute to a state of unease or generalized dissatisfaction with their biological sex. These point to a possible biological and genetic underpinning of GD as stemming from a discordance between gonadal and brain development. However, not enough evidence has associated these differences with GD. Further research is required to elucidate the true mechanisms and possible inheritance pattern of GD for a better education and greater understanding by clinicians and the general public on perceptions regarding GD.

\section{Plain Language Summary}

Gender identity is an important issue in society, yet its causative mechanism is poorly understood. The main anatomical differences between males and females are the genitalia and the brain. This paper reviewed the literature to explore the link between genetic influences and brain development, and their impact on gender identity. Investigating these developmental mechanisms could lead to advances in the understanding of gender dysphoria, a condition whereby an individual's gender and biological sex are mismatched. Exploring the biochemical development of the genitalia highlights the differences between males and females, notably how testosterone elicits the pathways of male development in an embryo. When research has been conducted in people with Androgen Insensitivity Syndrome, a condition where the testosterone receptor is mutated and faulty, and thus cannot function, gender dysphoria is observed as the body is genetically male but anatomically female.It is known that the structure of male and female brains differs; it is found that people with gender dysphoria have a brain structure more comparable to the gender to which they identify. The review of the literature suggests that there is a disparity between the brains of those who identify differently to their assigned gender at birth, highlighting a multifactorial underpinning of the gender identity. Further research is required to shed light on the molecular mechanism of this, allowing for greater education and understanding of this scientific and social phenomenon.

The Endocrine Society. Transgender Health An Endocrine Society Position Statement 202012 https://www.endocrine.org/advocacy/position-statements/transgender-health (The Endocrine Socieity, 2020)

Abstract : There is a durable biological underpinning to gender identity that should
be considered in policy determinations . Medical intervention for transgender youth and
adults (including puberty suppression, hormone therapy and medically indicated surgery) is ef-
fective, relatively safe (when appropriately monitored), and has been established as the standard 
of care. Federal and private insurers should cover such interventions as prescribed by a physician as well as the appropriate medical screenings that are recommended for all body tissues that a person may have. Increased funding for national pediatric and adult transgender health research programs is needed* to close the gaps in knowledge regarding transgender medical care and should be made a priority.

*NOTE: Remember what I said about how studies always or almost always will say further study is needed. This one is pretty upfront about why. We need money.

Amir, Hadar / Perl, Liat / Barda, Shimi / Lantsberg, Daniel / Becker, Anat Segev / Israeli, Galit / Azem, Foad / Oren, Adolescent Transgender Females Present Impaired Semen Quality That Is Suitable for Intracytoplasmic Sperm Injection Even Before Initiating Gender-Affirming Hormone Treatment 2022 Reproductive Sciences, Vol. 29 p. 260-269 https://doi.org/10.1007/s43032-02100561-y (Amir et al., 2022)

\begin{abstract}
The present study aimed to determine the semen quality and cryopreservation outcomes among adolescent transgender females at the time of fertility preservation (FP) before initiating gender-affirming hormone (GAH) treatment. This retrospective cohort study included 26 adolescent transgender females who underwent FP in our Fertility Institute between 06/2013 and 10/2020. Pre-freezing semen parameters were compared to WHO 2010 reference values. Postthaw semen parameters were used to determine the adequate assisted reproductive technology (ART). A multivariate linear regression analysis was performed to assess the impact of medical and lifestyle factors on semen quality. The mean age at which adolescent transgender females underwent FP was $16.2 \pm 1.38$ years. The median values of all semen parameters in our study group were significantly lower compared to the WHO data, including volume $(1.46 \mathrm{~mL}$ vs 3.2 $\mathrm{mL}$, respectively, $\mathrm{P}=0.001)$, sperm concentration $(28 \times 106 / \mathrm{mL}$ vs $64 \times 106 / \mathrm{mL}, \mathrm{P}<0.001)$, total sperm number $(28.2 \times 106$ vs $196 \times 106, \mathrm{P}<0.001)$, total motility $(51.6 \%$ vs $62 \%, \mathrm{P}<$ $0.001)$, and normal morphology $(2 \%$ vs $14 \%, \mathrm{P}<0.001)$. The frequency of semen abnormalities was teratozoospermia $72 \%$, hypospermia $52 \%$, oligozoospermia $28 \%$, and azoospermia $4 \%$. The median post-thaw total motile count was $0.17 \times 106 /$ vial, and the quality was adequate only for ICSI in $87.7 \%$ of the thawed semen samples. No correlation was found between selected medical and lifestyle factors and poor semen parameters. Semen quality is strongly reduced among adolescent transgender females before hormone therapy and their stored sperm samples are suitable for intracytoplasmic sperm injection (ICSI) rather than conventional $\mathrm{IVF} /$ intrauterine insemination (IUI).
\end{abstract}

\title{
Findings in sports medicine.
}

Hilton, Emma N. / Lundberg, Tommy R. Transgender Women in the Female Category of Sport: Perspectives on Testosterone Suppression and Performance Advantage2020 Sports Medicine https://doi.org/10.1007/s40279-020-01389-3 (Hilton \& Lundberg, 2020)

Abstract: Males enjoy physical performance advantages over females within competitive sport. The sex-based segregation into male and female sporting categories does not account for transgender persons who experience incongruence between their biological sex and their experienced gender identity. Accordingly, the International Olympic Committee (IOC) determined criteria by which a transgender woman may be eligible to compete in the female category, requiring total serum testosterone levels to be suppressed below $10 \mathrm{nmol} / \mathrm{L}$ for at least 12 months prior to and during competition. Whether this regulation removes the male performance advantage has not been scrutinized. Here, we review how differences in biological characteristics between biological males and females affect sporting performance and assess whether evidence exists to support the assumption that testosterone suppression in transgender women removes the male performance advantage and thus delivers fair and safe competition. We report that the performance gap 
between males and females becomes significant at puberty and often amounts to $10-50 \%$ depending on sport. The performance gap is more pronounced in sporting activities relying on muscle mass and explosive strength, particularly in the upper body. Longitudinal studies examining the effects of testosterone suppression on muscle mass and strength in transgender women consistently show very modest changes, where the loss of lean body mass, muscle area and strength typically amounts to approximately $5 \%$ after 12 months of treatment . Thus, the muscular advantage enjoyed by transgender women is only minimally reduced when testosterone is suppressed. Sports organizations should consider this evidence when reassessing current policies regarding participation of transgender women in the female category of sport.

The British medical journal conducted a large study of people who began hormone therapy as adults, female to male and male to female. While two years of cross sex hormone therapy did reduce muscle strength and mass a speed advantage was retained. Hilton EN and Lundberg T R published a paper which was well cited that stated a much larger advantage, but this was latter corrected by the journal due to undeclared conflict of interest.

Note in the following subsection, as the science is softer all findings pro and against trans inclusion bear a degree of opinion and point of view. The above chemical (DNA) and MRI and other laboratory findings do not have that issue.

Roberts, Timothy A. / Smalley, Joshua / Ahrendt, Dale, Effect of gender affirming hormones on athletic performance in transwomen and transmen: implications for sporting organisations and legislators, 2021 British Journal of Sports Medicine, Vol. 55, No. 11 British Association of Sport and Excercise Medicine p. 577-583 https://doi.org/10.1136/bjsports-2020-102329 (Roberts et al., 2021)

\begin{abstract}
Objective To examine the effect of gender affirming hormones on athletic performance among transwomen and transmen.Methods We reviewed fitness test results and medical records of 29 transmen and 46 transwomen who started gender affirming hormones while in the United States Air Force. We compared pre- and post-hormone fitness test results of the transwomen and transmen with the average performance of all women and men under the age of 30 in the Air Force between 2004 and 2014. We also measured the rate of hormone associated changes in body composition and athletic performance.Results Participants were 26.2 years old (SD 5.5) . Prior to gender affirming hormones, transwomen performed $31 \%$ more push-ups and $15 \%$ more sit-ups in 1 min and ran 1.5 miles $21 \%$ faster than their female counterparts. After 2 years of taking feminising hormones, the push-up and sit-up differences disappeared but transwomen were still $12 \%$ faster. Prior to gender affirming hormones, transmen performed $43 \%$ fewer push-ups and ran 1.5 miles $15 \%$ slower than their male counterparts. After 1 year of taking masculinising hormones, there was no longer a difference in push-ups or run times, and the number of sit-ups performed in $1 \mathrm{~min}$ by transmen exceeded the average performance of their male counterparts.Summary The $15-31 \%$ athletic advantage that transwomen displayed over their female counterparts prior to starting gender affirming hormones declined with feminising therapy. However, transwomen still had a 9\% faster mean run speed after the 1 year period of testosterone suppression that is recommended by World Athletics for inclusion in women's events . A de-identified copy of the data is available from the corresponding author upon reasonable request.
\end{abstract}

Hilton, Emma N. / Lundberg, Tommy R. CORRECTION To: Transgender Women in the Female Category of Sport: Perspectives on Testosterone Suppression and Performance Advantage 2021 Sports Medicinehttps://doi.org/10.1007/s40279-020-01389-3 (Hilton \& Lundberg, 2021)

Abstract: After publication of this article, concerns were raised regarding potential undeclared
conflicts of interest . In light of this the authors have provided the following statement: EH
and TL have given talks and engaged in the mainstream media and academic press regarding 
the biology of sex and how they have concluded that this should impact sporting categories. All dissemination or engagement, irrespective of the medium, has been guided by their education, disciplinary training, and research findings. The authors assert that this does not constitute a conflict of interest. Rather, it is an essential part of their academic freedom and their obligation to engage, publicly, in such discussions.

(See for example: Harder, better, faster, stronger: why we must protect female sports by Hilton. "Male puberty and testosterone. Testosterone, the androgen driving male physical development, is a wonderful hormone. It is responsible for advantageous skeletal features that develop during male puberty, such as increased height, increased bone size and density, longer limbs," In short in a field of science where one can select data in such a way as to create a certain result she selected top tier athletes and only transgender people who transitioned as adults, post puberty not during puberty age. The second point is a weakness of all of the studies in this section and seems to be a standard practice. Which is why I searched out the British Association of Sports Medicines unconflicted study that partially agrees with hers. This leaves open the question what of transgender people who started hormones very young and who have little or no advantage or disadvantage?) This researchers mind was totally made up before she started, and she also did not disclose this fact to the reviewers.

\section{British Journal of Sports Medicine, Plain language findings and expert opinion.}

Testosterone and Transgender Athletic Performance : Finding a path for inclusion for transgender athletes by BJSM https://blogs.bmj.com/bjsm/2021/01/22/testosterone-and-transgender-athleticperformance-finding-a-path-for-inclusion-for-transgender-athletes/ (Ahrendt et al., 2021)

Transgender individuals have been able to serve openly and have access to gender affirming hormone therapy in the United States military since 2016. In the Air Force, requests to begin or continue hormonal therapy are reviewed by a centralized clinic. Of the 222 transgender service members who requested permission to start or continue treatment, we had complete fitness and medical data on 46 transwomen and 29 transmen. The average age was 26.2 with a standard deviation of 5.5 years.

For transmen, there was no significant change in body composition (as measured by height, weight, and waist circumference) over time. They were able to perform the same number of situps as cismen prior to hormone therapy and improved with therapy. Push-ups and run times reached that of cismen by the 1-year point.

For transwomen undergoing hormone therapy, there was an increase in weight over time but no change in waist circumference. Push-ups and sit-ups equalized with ciswomen by the 2-year mark. However, transwomen still retained an advantage in run times on the mile and a half run compared to the average time for ciswomen $2 \frac{1}{2}$ years after starting medical transition

\section{What does this mean?}

In elite level or collegiate athletic competition, where a $1-2 \%$ advantage in speed or strength is often the difference between victory and defeat, governing bodies need to re-examine guidelines for inclusion of transgender and other women with elevated testosterone levels and determine what evidence-based changes are required to ensure a level playing field. They will need to stand up to legal scrutiny. In youth and recreational sports there is such a broad range of ability, training, and development that differences from prior testosterone after 12 months of suppression may not have a meaningful competitive impact and the benefits of inclusion of all athletes should take priority. When establishing guidelines for youth and recreational sports, the question has to be: "What is the best thing for all our athletes?"

\section{Legal and policy opinions.}

IF one considers themselves liberty minded these are the opinions of liberty minded people. Meaning rights 
for other people not just a majority of people.

The Libertarian Party of the United States of America. Political Stance Attacks on Trans people are an attack on Liberty 2021 https://www.lp.org/attacks-on-trans-people-are-an-attack-onliberty/ (of the United States of America, 2021)

Statement: There are some who will always find true Liberty for all to be too risky an idea to embrace - unfortunately, many of those people are elected officials. In state legislatures across the country, bills have been proposed (and unfortunately in some cases, passed) that would regulate and criminalize the personal medical and life decisions of transgender individuals. Those who long have declared themselves champions of limited government and personal freedom are seeking to insert their own opinions into decisions that should be left to individuals and families.

In Arkansas where some bills have already become law after legislators overrode a veto, Governor Hutchinson expressed the same confusion so many share. "The Republican Party that I grew up with believed in a restrained government that did not jump in the middle of every issue." Of course, anyone paying attention has recognized that neither old party is interested in a restrained response to any concern.

As is the only apparent tactic of status quo politicians these days, those championing anti-liberty bills are spreading fear, lies, and sensational stories to secure support for limiting the rights of those they simply do not understand or do not want seen as acceptable. In Texas, there is an effort to classify gender-affirming care as child abuse which can be classified as a felony with corresponding jail time. Right now there are parents wondering if they will have to uproot their families and flee their home state rather than neglect the carefully considered care that is helping their children thrive.

There is perhaps nothing more dangerous to individuals and families than elected officials who value their own opinions over the love and devotion of a parent seeking expert opinions and the best life for their child.

Buzuvis, Erin Law, policy, and the participation of transgender athletes in the United States 2021 Sport Management Review, Vol. 24, No. 3 Routledge p. 439-451 https://doi.org/10.1080/14413523. 2021.1880757 (Buzuvis, 2021)

Abstract: This Article provides an overview of United States law and public policy relating to the rights of transgender athletes to participate in sports programs. United States public law is complex but generally permissive of inclusion. The Author opines how sports organizers should apply the law, or in its absence, what to consider when creating internal policies, including that sports organizations should allow gender-identity based participation; participation restrictions are inappropriate in youth, non-elite athletics; and inclusion promotes dignity, respect and health of transgender participants.

Webb, Karleigh New research paints a more complex picture of transgender sports advantage Updated Mar 10, 2021, 10:21am PST https://www .outsports.com/2021/3/9/22321015/joanna-harpertransgender-athlete-research (Webb, 2021)

For those who suggest trans women have advantages: we allow advantages in sport, but what we don't allow is overwhelming advantages," she said. "Trans women also have disadvantages in sport. Our larger bodies are being powered by reduced muscle mass and reduced aerobic capacity, and can lead to disadvantages in quickness, recovery and a number of other factors.

"The bottom line is, we can have meaningful competition between trans women and cis women. From my point of the view, the data looks favorable toward trans women being allowed to compete in women's sports. 
NOTE: This is the opinion of a researcher on the unconflicted article in the British Sports Medicine Journal. Unconflicted because the author declared and did not hide her possible bias and worked with a large team that would not share that. NOTE even the author of the conflicted paper cites this transwomans work as being high quality.

She cautions that all studies on the issue, even her groundbreaking work in 2015 that first compared a small section of trans women distance runners with each other, has to be placed in a larger context surrounding the discussion of sports.

\section{Summary of Conclusions and Findings.}

The above are peer reviewed and published data and expert findings of fact the below is my summary of them:

Multiple independent studies have demonstrated a high probability that mutations to the Androgen Receptor (AR) and estrogen receptor genes ER $\beta$ (Fernández et al., 2018) (Theisen et al., 2019) These genetic differences correspond to various differences in brain strucutre such that sexually dimorphic brain structures of transwomen match those of ciswomen, and those of transmen match those of cismen. (Boucher \& Chinnah, 2020) The differences are also mannifested in the form of reduced fertility and general undermasculinization even prior to any hormone treatment. (Amir et al., 2022) The above finding is backed by MULTIPLE independent expert, peer reviewed, published meta analysis, a study of all available studies. (D'Andrea et al., 2020) Crucially for the issue of fairness in sports there are relevant endocrinological differences. (The Endocrine Socieity, 2020) A study done on post pubescent transgender adults by (Hilton \& Lundberg, 2020) concluded a large advantage for transgender women in every sport, however, this article was corrected by the journal due to their undisclosed confict of interest. (Hilton \& Lundberg, 2021) A further study, also cited by Hilton as being well done, found after 1-2 years on hormones little to no difference in strength but a $5 \%$ advantage in speed for transwomen. (Roberts et al., 2021) Based on (Roberts et al., 2021) the British Journal of Sports Medicine concluded that given the data on competitors who are transitioning as young adults at the elite and collegiate levels there can be a difference that confers advantage, at the same time at the high school, youth and recreational levels when all the data is considered there is not necessarily an advantage. (Ahrendt et al., 2021)

In the opinion of a trans researcher on the team who worked on (Roberts et al., 2021) which was cited by the British Journal of Sports medicine, (Ahrendt et al., 2021), the issue of sports is nuanced with advantage in speed but not necessarily in strength over time. (Webb, 2021)

The Policy opinion of the Libertarian Party of the United States of America, hardly a left wing party "Attacks on Trans people are an attack on Liberty" that fear, lies, and sensational stories are being used to justify taking rights from a easily villified minority, and reduce the rights of the majority in the process." (of the United States of America, 2021)

According to one Sports Mangement law review, something which the author of the current document admits is not in her area of expertise the current public laws of the United States lean towards inclusivity. (Buzuvis, 2021)

Given the scientific facts as confirmed by multiple studies and taking the biologically essentialist framework as a given we can answer the key questions.

Do transgender people have the choice of completely conforming to gender norms and just being manly men or feminine women? Does the available data show that transgender people exist due to genetic, hormonal, neurological and or any other physical and immutable cause?

Being transgender in the broadest sense is not a choice and has an immutable cause that lay in genetics and hormonal influences on the structure of the brain. (Fernández et al., 2018) (Theisen et al., 2019)(Boucher \& Chinnah, 2020)(Amir et al., 2022) (D'Andrea et al., 2020) (The Endocrine Socieity, 2020) Transgender 
people make choices on how to handle this with the guidance of the doctors, psychologist, and medical experts (of the United States of America, 2021). They are no more obligated to conform to anyone elses expectations to be deserving of rights.

Sports is one area where the rights of transwomen may have a persistent advantage. Does hormone therapy for enough time or given early enough in life level the playing field for sports, and what variables effect this? The answer is when it comes to, young adult transitioners muscular strength, after 1-2 years on hormones, most differences will vanish. Transgender women do retain an advantage in speed after that period. (Roberts et al., 2021) At the level of youth elementary, middle and high school sports due to the differential effcts of puberty someone changing at those ages will have no material advantage (Ahrendt et al., 2021). Further research is needed into the advantages if any at the level of elementary school, and high school.

\section{References}

Molecular basis of Gender Dysphoria: androgen and estrogen receptor interaction. (2018). Psychoneuroendocrinology, 98, 161-167. https://doi.org/10.1016/j.psyneuen.2018.07.032

The Use of Whole Exome Sequencing in a Cohort of Transgender Individuals to Identify Rare Genetic Variants. (2019). Nature Scientific Reports, 9, 20099. https://doi.org/10.1038/s41598-019-53500-y

Gender Dysphoria: A Review Investigating the Relationship Between Genetic Influences and Brain Development. (2020). Adolescent Health, Medicine and Therapeutics, Volume 11, 89-99. https://doi.org/10. 2147 /ahmt.s259168

Adolescent Transgender Females Present Impaired Semen Quality That Is Suitable for Intracytoplasmic Sperm Injection Even Before Initiating Gender-Affirming Hormone Treatment. (2022). Reproductive Sciences, 29, 260-269. https://doi.org/10.1007/s43032-021-00561-y

Polymorphic Cytosine-Adenine-Guanine Repeat Length of Androgen Receptor Gene and Gender Incongruence in Trans Women: A Systematic Review and Meta-Analysis of Case-Control Studies. (2020). The Journal Of Sexual Medicine, 17, 543-550. https://doi.org/10.1016/j.jsxm.2019.12.010

Transgender Health An Endocrine Society Position Statement. (2020). The Endocrine Society. https: //www.endocrine.org/advocacy/position-statements/transgender-health

Transgender Women in the Female Category of Sport: Perspectives on Testosterone Suppression and Performance Advantage. (2020). Sports Medicine. https://doi.org/10.1007/s40279-020-01389-3

CORRECTION To: Transgender Women in the Female Category of Sport: Perspectives on Testosterone Suppression and Performance Advantage. (2021). Sports Medicine. https://doi.org/10.1007/s40279020-01389-3

Effect of gender affirming hormones on athletic performance in transwomen and transmen: implications for sporting organisations and legislators. (2021). British Journal of Sports Medicine, 55(11), 577-583. https://doi.org/10.1136/bjsports-2020-102329

Testosterone and Transgender Athletic Performance : Finding a path for inclusion for transgender athletes. (2021). British Journal of Sports Medicine (Official Blog). https://blogs.bmj.com/bjsm/2021/01/ 22/testosterone-and-transgender-athletic-performance-finding-a-path-for-inclusion-fortransgender-athletes/

Attacks on Trans people are an attack on Liberty. (2021). Libertarian Party Of The United States Platform. Law, policy, and the participation of transgender athletes in the United States. (2021). Sport Management Review, 24(3), 439-451. https://doi.org/10.1080/14413523.2021.1880757 
New research paints a more complex picture of transgender sports advantage. (2021). Out Sports. 